\title{
Morphological Studies of the Oral Roof of the Egyptian Laughing Dove (Streptopelia senegalensis aegyptiaca) and Japanese Quail (Coturnix coturnix japonicum)
}

\author{
Fatma A. Madkour
}

Department of Anatomy and Embryology, Faculty of Veterinary Medicine, South Valley University, Qena 83523, Egypt

Received September, accepted for publicatioin October 2018

\section{Abstract}

This study aimed to determine the morphological characteristics of the palate, choanal slit of two birds of different families; Japanese Quail (JQ) for family Phasianidae and Laughing Dove (LD) for family Columbidae by gross anatomy, scanning electron microscopy and light microscopy. The organs of 16 birds (eight laughing doves and eight Japanese quails) were used. The length of the oropharyngeal roof was nearly equal in both birds; it was divided into oral and pharyngeal roofs. The oral roof (palate) was narrow elongated triangular shaped in LD and wide triangular shaped in JQ, and the oral roof (palate) was longer in LD than that of JQ. The ratio of the oral roof (palate) to the total length of the oropharynx was $86.36 \%$ in LD and $72.82 \%$ in JQ. The choanal slit constituted a nearly same percentage of the length of the palate which was $49-50 \%$ while to the total length of the oropharynx was $43.19 \%$ in LD and $36.75 \%$ in JQ. The line of separation between narrow and wide parts of the choanal slit demarcated by caudolaterally directed papillae and mucosal elevation in LD and by a transverse row of $\mathrm{V}$-shaped papillae in JQ. The edges of the rostral narrow part bear small sized widespaced papillae having serrated appearance, while the edges of the wide part were smooth in LD. In JQ 3 transverse rows of papillae and one transverse mucosal fold were presented around the choanal slit. The lamina propria of the palate consisted of dense connective tissue, rich in several types of sensory corpuscles; Merkel's corpuscles were the majority of the sensory corpuscles in LD. Herpst corpuscles with variable sizes and shapes were the majority of sensory corpuscles 
in JQ. Submucosa of the palate on each side of the choanal slit consisted of 2 groups of palatine salivary glands; medial and lateral groups in $\mathrm{JQ}$ and few lobules of the medial salivary glands were observed in LD.

\section{Keywords}

scanning electron microscopy; light microscopy; oral roof, Laughing Dove, Japanese Quail.

\section{Introduction}

Laughing dove is a common and prevalent species in scrub, dry farmland, and housing over a good deal of its range, often becoming very domesticated. The species is found in much of Sub-Saharan Africa, Saudi Arabia, Iran, Afghanistan, Pakistan, and India. It is also found in Israel, Lebanon, Syria, UAE, and Turkey (Ticehurst, 1923, Ali and Ripley, 1981). The laughing doves feed on seeds, grasses, other vegetable matter and small ground insects such as termites and beetles (Satheesan et al., 1990, Adang et al., 2008). Inhabitance of the Japanese quail are known to mainly inhabit East Asia and Russia. This includes India, Korea, Japan, and China (Barilani et al., 2005, Puigcerver et al., 2007, Pappas, 2013). And this species has also been found to banquet in many parts of Africa, including Tanzania,
Malawi, Kenya, Namibia, Madagascar, and the area of the Nile River Valley extending from Kenya to Egypt (Pappas, 2013). The Japanese quail is primarily a ground-living species that tends to stay within areas of dense vegetation in order to take cover and evade predation (Buchwalder and Wechsler, 1997). Thus, its natural habitats include grassy fields, shrubs along the banks of rivers, and agricultural fields that have been planted with crops such as oats, rice, and barley (Buchwalder and Wechsler, 1997, Pappas, 2013).

Birds have different feeding habits with corresponding differences in the structure of their oropharyngeal cavity, so the anatomy of the avian oropharyngeal cavity is important to identify the structural variations that may influence nutrition, food intake, and ingestion (Jayachitra et al. 2015). The movement of the bolus toward the pharynx would appear to be facilitated by the caudally directed papillae on the tongue and the palate (McLelland, 1975, King and McLelland, 1984). The mucosal lining of the oral cavity and esophagus is stratified squamous epithelium (masticatory mucosa), functioned to protect the underlying tissue from mechanical damage and from entry of micro-organisms and 
toxic materials that may be presented in the oral cavity and the oropharynx (Squier and Kremer, 2001).

The study on the roof of the oropharynx of the laughing doves and Japanese quails was inadequate and I did not find sufficient information about this point. The objective of this study was, therefore, to describe grossly, morphometrically and SEM of the oral roof of these birds which have different feeding habits and comparing the study with the results with previous data from other birds obtained from the literature.

\section{Material and methods}

\section{I- Sampling}

Eight adults healthy LD (94.26 $\pm 8.02 \mathrm{gm}$ ) were collected from bird hunters and eight adults healthy $\mathrm{JQ}$ $(235.67 \pm 2.5 \mathrm{gm})$ were obtained from researcher's farm in South Valley University, Qena governorate, Egypt. The birds were sacrificed and complete bleeded, then heads were dissected and washed with distilled water and then saline. The present work was done following the guidelines of the Institutional Ethical Committee.

\section{II- For gross morphology and morphometrical measurements}

Five heads of each bird were used. The oropharynx was opened at the

J. Vet. Anat. angle of the mouth; its roof was dissected and fixed in 10\% neutral buffered formalin. The various gross morphological features for each bird separately were examined in detail by the naked eyes and magnification lens and photographed by a digital camera (Panasonic DMC-F3). The different measurements in millimeters (mean \pm S.E.) of the studied parts; oral roof (palate), choanal slit were taken out using Precision Digital Vernier Caliper.

\section{III- Sampling processing for scanning electron microscopy}

One head of each bird was used. Representative specimens from rostral and caudal parts of the palate and choanal slit were washed several times with normal saline and then fixed in a mixture of $2.5 \%$ paraformaldehyde and $5 \%$ glutaraldehyde in $0.1 \mathrm{M}$ sodium phosphate buffer, $\mathrm{pH} 7.3$, at $4^{\circ} \mathrm{C}$ for 24 hours. Thereafter, they were washed 4 times for $5 \mathrm{~min}$ in the fixation buffer and postfixed in $1 \%$ osmic acid in $0.1 \mathrm{M}$ sodium phosphate buffer for further 2 hours at room temperature, followed by washing with $0.1 \mathrm{M}$ sodium phosphate buffer for 15 min 4 times. The samples were dehydrated using increasing concentrations of alcohol: 50,70 , and $90 \%$ for 30 min at each concentration and $100 \%$ for 2 days (several changes) followed by isoamyl acetate for 2 days. The dehydrated samples were 
subjected to critical point drying with a Polaron apparatus. Finally, they were coated with gold using JEOL$1100 \mathrm{E}$ ion sputtering device and examined with a JEOL scanning electron microscope (JSM 5500 LV) at $10 \mathrm{kV}$.

\section{IV- Sampling processing for light microscopy}

Two heads of each bird were used. Oral roof (palate), and choanal slit were washed then fixed in $10 \%$ neutral buffered formalin. After proper fixation, the samples were kept in formic acid for the process of decalcification. After decalcification, the specimens were washed at least 6 to 12 hours under running tap water and then dehydrated in ascending grades of alcohol. The dehydrated samples were cleared in methyl benzoate for 24 hours and embedded in paraffin wax. Serial sections (5- $\mu \mathrm{m}$ thickness) were obtained on a LEICA 2165 microtome. The paraffin sections were dewaxed and rehydrated in a descending series of ethanol and were subjected to the following stains: Harris hematoxylin and eosin (H\&E), Masson's trichrome, Periodic-Acid Schiff (PAS).

\section{V- Statistical analysis}

All measurements were statistically analyzed by the Statistical Package for Social Science (SPSS) software program, version 17.0 (Argyrous, 2005).

J. Vet. Anat.

\section{Result}

\section{Gross anatomical \& morpho- metrical investigation}

The oropharyngeal roof could be divided into oral and pharyngeal parts. The demarcation between 2 parts lied between choanal and infundibular slits. The line of separation between the two parts was located behind the level of the angle of the mouth by nearly same distance in both birds $5.71 \mathrm{~mm}$ in LD and $5.74 \mathrm{~mm}$ in JQ. The oral roof is occupied by the palate which is presented by a median cleft called choana (choanal slit), which connects the oral nasal cavities. The palate was longer in LD than that in JQ. It measured $23.30 \mathrm{~mm}, 20.15 \mathrm{~mm}$ constituted $86.36 \%$, and $72.82 \%$ of the total length of the oropharynx in LD and JQ respectively. The palate was narrow elongated triangular shaped in LD and wide triangular shaped in $J Q$. The palate increased gradually in its width caudally (Fig1A, B). The width of the palate in LD was 2.01, $4.94,6.43 \mathrm{~mm}$, while in JQ was $2.95,8.65,10.63 \mathrm{~mm}$ at the level of tip of the beak, narrow and wide parts of choanal slit respectively.

The palate could be divided into two parts, rostral and caudal; the line of division was demarcated between the angles of the mouth. This line lied at the junction of the narrow rostral and wide parts of the choanal slit, therefore, the rostral part of the 
palate contained the narrow part while the caudal part of the palate contained the wide part of the choanal slit.

The rostral part of the palate is characterized by 3 palatine ridges; one median and two lateral palatine ridges. The median palatine ridge extended behind the tip of the beak by $2 \mathrm{~mm}$ in $L D$ and $3.81 \mathrm{~mm}$ in JQ. The median palatine ridge in LD was thick and prominent rostrally and decreased gradually in thickness caudally and terminated at the rostral end of the choanal slit and continued by a median septum. The two lateral palatine ridges extended nearly rostrolaterally from both sides of the middle part of the median palatine ridge and continued caudally along the palate terminated at the junction between oral and pharyngeal roofs. Moreover,these ridges become more prominent caudally resulting in two shallow palatine grooves between these ridges and the choanal slit (Fig.1A). In JQ, the median palatine ridge increased in thickness caudally and terminated at junction with two lateral palatine ridges by a distance in front of the choanal slit and the two lateral palatine ridges began by termination of the median ridge which decreased in thickness caudally and terminated at the transverse row of papillae between the narrow and wide parts of the choanal slit (Fig.1B).
The choanal slit was a median slit located just rostral to the infundibular slit. It consisted of long narrow rostral and short wide caudal parts. The narrow part is located at the rostral part of the palate while the wide part is located at the caudal part of the palate. In LD, the line of demarcation between the narrow and wide parts is marked by caudolaterally directed papillae and mucosal elevation on each side. The edges of the narrow part of the choanal slit bear few fine papillae while of the wide part were smooth and free from papillae (Fig.1A). In JQ, the line of demarcation between the two parts is marked by a transverse row of papillae. Grossly, three transverse rows of papillae and one transverse mucosal fold were distributed around the choanal slit. One row presented in front of the choanal slit behind the junction between the palatine ridges and the second row at the rostral end of the slit and the mucosal fold on each side of the narrow part of the choanal slit. The third transverse row is situated at the junction between the narrow and wide parts. Moreover, irregular distribution of papillae on each side of the slit and caudoventrally directed papillae were observed on edges of the choanal slit (Fig.1B). The statistical data clarified that the length of the choanal slit measured $11.52 \mathrm{~mm}$, $10.17 \mathrm{~mm}$ constituted $43.19 \%, 36.75$ $\%$ of the total length of the oropharJ. Vet. Anat. 
ynx and $49.44 \%, 50.57 \%$ of the palate in $\mathrm{LD}$ and $\mathrm{JQ}$ respectively. The length of the narrow part was $7.38 \mathrm{~mm}, 6.13 \mathrm{~mm}$, constituted 64.24 $\%, 60.28 \%$, and the wide part was $4.12 \mathrm{~mm}, 4.04 \mathrm{~mm}$, constituted 35.76 $\%, 39.72 \%$ of the total length of the choanal slit in LD and JQ respectivly

\section{Scanning electron microscopy}

The scanning electron microscope examination of the palate of LD revealed that the median and lateral palatine ridges were separated from each other by 2 shallow grooves. The caudal part of the median palatine ridge was interrupted before its termination and continuation with the median septum at the choanal slit. Small few papillae were observed between the caudal part of the median and the lateral palatine ridges.

By higher magnification on either side of the caudal part of the median palatine ridge two openings of the maxillary salivary glands were demonstrated (Fig. 2A, C, E). In JQ, two elongated mucosal folds were observed on each side of the median palatine ridge. Three caudally directed papillae with a wide base and pointed apices were observed caudal to the junction between the three palatine ridges; the middle papilla was the largest one. Rostral to this row at the angle of the junction between the median and lateral ridges; two openings of the maxillary

J. Vet. Anat. salivary glands were scattered (Fig. 2B, D, F).

Concerning the narrow part of the choanal slit in LD, appeared as an elongated slit, its edges of the rostral part bear small sized wide spaced papillae which were indistinctly rostrally and became more distinct and larger caudally, these papillae giving the edges of the slit a serrated appearance. Additionally, three caudally directed with pointed apices papillae were demonstrated laterally to the edge of the rostral half of the narrow part and an elongated mucosal elevation was observed on each side of the caudal half, this elevation terminated by beginning the wide part (Fig. 3A, C). While in $J Q$, the narrow part of the slit was wider than that of LD. The transverse row of papillae at the rostral end of the narrow part consisted of five papillae; one median, conical in shape at the beginning of the median septum, two on the edges of the rostral end of the slit giving bulb appearance, in beside which two another papillae were present. The row on each side of the narrow part consisted of 4 papillae; 2 small sized on the edges of the slit and 2 large sized with a wide base and pointed apices on each side of the slit. The transverse mucosal fold on each side of the narrow part of the slit exhibited winglike shape extended from edges of the slit to the lateral edge of the pal-

Vol. 11, No. 2, (2018) 17 - 39 
ate. The row of papillae at the junction between the narrow and wide parts of the slit consisted of numerous caudally directed $V$-shaped papillae (Fig. 3B, D, F).

The edges of the wide part of the choanal slit in LD were smooth and free from papillae (Fig. 3E). Few small openings of palatine salivary glands were observed on each side of the caudal narrow part of the choanal slit (Fig. 4A, B). In JQ, the edges of the wide part bear conical shaped caudally directed papillae. Additionally, on each side of the wide part numerous different sizes and shape papillae; some of these papillae were conical shaped with pointed apices and the others were nodular in shape (Fig. 3F). Numerous openings of the medial palatine salivary glands were scattered between these papillae medial to the lateral palatine ridge which increased caudally. In addition, the lateral palatine salivary glands were observed on the lateral palatine ridges and also on palatine mucosa lateral to the lateral ridge (Fig. 4CF). Numerous of intraepithelial glands were observed within the epithelium of the choanal slit and these openings were different in size and shape in both birds (Fig. 3G, H).

\section{Light microscopy}

The mucosa of the palate consisted of a highly cornified stratified squamous epithelium. The cornification

J. Vet. Anat. increased in thickness laterally toward the edges of the beak. At the rostral part of the palate of $\mathrm{JQ}$ in addition to the median ridge and the two folds on each side of it; appeared another two folds laterally separated from the beak edges by grooves (Fig. 5A, B). The lamina propria was thickest at the level of the median palatine ridge, consisted of dense connective tissue, rich in blood vessels, collagenous fibers, moreover, several types of sensory corpuscles. In LD, the majority of the sensory corpuscles were Merkel's corpuscles which composed of sensory cells surrounded by a capsule, intraepithelial Merkel corpuscles were demonstrated within lining epithelium and few numbers of Herbst and Ruffini corpuscles. The Herbst corpuscles were oval to an elongated structures composed of central axon with sensory cells enclosed by fine collagen fibers and capsule, some of these corpuscles have two axons (Fig. $5 C)$. In JQ the majority of the sensory corpuscles were Herbst corpuscles with variable size and shape, some corpuscles consisted of sensory cells arranged in a row along the axon. Ruffini corpuscles were also observed in addition to Merkel corpuscles which were situated close to the basement membrane of lamina epithelialis (Fig. 6D).

Submucosa of the palate consisted of loose connective tissue contained 
large blood vessels, nerve trunks and two groups of tubuloalveolar maxillary salivary glands on both sides of the median palatine ridges in the rostral third of the rostral part of the palate which extended caudally rostral to the choanal slit, these glands were divided into lobules which consisted of several secretory tubules (Fig. 6A-D).

In LD, the lining epithelium of the secretory units of the glands was mucous secretory cells appeared as low columnar cells with faint stained foamy cytoplasm and rounded basally located nuclei, in addition, these glands were surrounded on both sides by large blood vessels with melanocytes around the glands capsule (Fig. 6C). In JQ, these glands were serous in type with few mucous secretory cells exhibited as cuboidal epithelium with basophilic stained cytoplasm and round basally located nuclei, these glands surrounding by a thin layer of smooth muscle fibers which squeezed on the glands for discharging the secretion (Fig. 6B\&D). In both birds, two nerve trunks and abundant adipose tissue were observed caudal to the maxillary salivary glands (Fig. $6 A \& B)$.

The lining epithelium of the palate at the level of the choanal slit was cornified stratified squamous epithelium in type, continued for a long dis- tance at the level of the narrow part in $L D$, short distance in JQ before transforming into pseudostratified ciliated columnar epithelium (Fig. 7A, C, D). The lining epithelium at the wide part of the latter bird was highly cornified with variable sizes of papillae appeared on each side of this part. These papillae were consisted of core of connective tissue covered with highly keratinized stratified squamous epithelium (Fig.8A).

The respiratory epithelium was interrupted by intra-epithelial glands which were seromucoid in type in LD; mucous secretory cells were columnar cells with foamy cytoplasm and serous secretory cells were cuboidal cells with rounded basally located nuclei while in JQ these glands were of the mucous type, lining by low columnar epithelium with foamy cytoplasm and rounded basally located nuclei (Fig. 7B). The lamina propria consisted of dense connective tissue, blood vessels, small number of sensory corpuscles with little lymphatic infiltration at the narrow part in LD which increased caudally. In JQ excessive lymphatic infiltration extended to the transitional point of the epithelium, the intra-epithelial lymphatic infiltration surrounding the intraepithelial glands along the choanal slit (Figs. 7\&8). The Submucosa consisted of two groups of palatine salivary glands, medial and lateral, surrounded by smooth muscle fibers in 
JQ which extended longitudinally on each side of the choanal slit, the medial group was more numerous than the lateral one. These glands were compound tubuloalveolar surrounded by a connective tissue capsule from which connective tissue septa dividing these glands into lobules were detached (Fig. 7D-F). The glands were mostly of the mucous type, showed positive reaction to PAS technique. The ducts of these glands were lined by cuboidal cells with basally located rounded nuclei and basophilic stained cytoplasm. These cells showed negative reactions for PAS technique (Fig. 8A-C).

In LD, few lobules of the medial salivary glands were of the mucous type with a thick layer of collagen fibers on each side of the caudal half of the narrow part of the slit (Fig.7C). In the same bird, the respiratory epithelium at the wide part was interrupted by mucous intraepithelial glands and showed positive reaction wih PAS technique. In addition, plate of maxillary bone were observed at the submucosa of this part (Fig. 8D\&E). The median septum at the narrow part of the slit of LD was lined by respiratory epithelium. In JQ this septum was not completely lined by respiratory epithelium but lined by stratified squamous epithelium at the center. Moreover, the mucous glands were demonstrated within lamina propria of the median septum at the level of wide part of the choanal slit (Figs. 7D\&8A).

In conclusion, this study documented that there were difference in shape of the palate and the number of palatine papillae around the choanal slit. Where there were several rows of the palatine papillae in $\mathrm{JQ}$ and few distribution of the papillae in $L D$, this refers to the different habits of the two birds, where JQ was ground bird eat with rapacity more than LD which was flying bird; the papillae obstruct the slit and facilitate the movement of food into the esophagus.

\section{Discussion}

From the current morphometrical study, it was revealed that the percentage of the length of the oral roof (palate) to that of the oropharyngeal roof was $86.36 \%$ in LD and $72.82 \%$ in JQ. Nearly like our findings in LD mentioned in turkey (Sayed et al., 2016). Mohamed and Zayed (2003) stated that the palate forms about $78 \%$ of the total length of the oropharynx in the pigeon and goose, but in the chicken, it forms only $67 \%$, while in duck, it was $84.47 \%$ (Madkour, 2011).

It was obvious from the present findings that the palate could be divided into two parts, rostral and caudal; the line of division lies at the junction of the narrow and wide parts of the choanal slit. Similar to that given in 
chicken and pigeon (Mohamed and Zayed, 2003). On contrary to the description of Rossi et al. (2005) in partridge and Abumandour (2014) in falcon mentioned that the rostral part of the palate lies rostral to the choanal slit, while the caudal part containing the slit. Moreover, in muscovy duck, two transverse ridges that demarcate the caudal aspects of the hard palate from the choanal slit (Igwebuike and Anagor, 2013).

The present study showed that the palate characterized by 3palatine ridges; one median and 2lateral ridges. These findings somewhat simulate the statement of Mohamed and Zayed (2003), Nickle et al. (1977) in chicken and pigeon and Jayachitra et al. (2015) in Guinea fowl. In this connection, the palate of the goose has a median longitudinal palatine ridge and 2-3 paramedian rows (King and McLelland, 1984, Mohamed and Zayed, 2003). While in emu only a prominent median palatine ridge is present and the lateral palatine ridges are absent (Crole and Soley, 2011) and (Tivane et al., 2011). Moreover, our results in LD somewhat simulate results of Mohamed and Zayed (2003) in pigeon that the palate characterized by longitudinal grooves that may be used as a passage to direct grains toward the pharynx. A groove between the lateral palatine ridges and tomium of the beak also helps to grip the closure in LD and JQ as stated by Ziswiler (1965) in birds. As shown by SEM in LD, the median longitudinal ridge in turkey interrupted at its caudal part (Sayed et al., 2016A). Corresponding to the scanning electron microscopical results, few openings of maxillary salivary glands are observed at the rostral part of the palate at the level of the caudal part of the median palate ridge. While Madkour (2011) claimed that two openings of the maxillary salivary glands were observed one opening on each side of the rostral part of the median longitudinal ridge. Mohamed and Zayed (2003) added in the goose that several small openings (maxillary) are demonstrated on either side of the median palatine ridge. And in the pigeon, four openings (maxillary) are demonstrated in the form of a longitudinal row on both sides of the median palatine ridge.

Our results recorded that the choanal slit constituted $49-50 \%$ of the total length of the palate in both studied birds. These percentages are somewhat like the statement of Mohamed and Zayed (2003) in the chicken, pigeon but unlike in goose which it constitutes $27.06 \%$ and it constituted about $34.98 \%$ in duck (Madkour, 2011). In this respect, the choanal slit of Guinea fowl measures $0.85 \pm 0.34 \mathrm{~cm}$ in males and $0.70 \pm 0.37 \mathrm{~cm}$ in females 
(Sundaram et al., 2015), 25ml in falcon (Abumandour, 2014). Tivane et al. (2011) and Rodrigues et al. (2012) noted that the choana in the ostrich is very short. Additionally, the statistical study of the present work showed that the wide part of the choanal slit constituted $35.76 \%$ in LD and $39.72 \%$ in JQ of the total length of the choanal slit. The percentage in JQ like the statement of Mohamed and Zayed (2003) in the chicken but it constitutes about $25 \%$ and $56.5 \%$ of the total length of the slit in pigeon and goose respectively. The wide part is double the narrow part of the duck (Hassouna, 2002). On other hand, the choanal slit in cattle egret was undivided (Moussa and Hassan, 2013).

Corresponding to the present findings, the edges of the narrow part of the choanal slit of LD bear few fine papillae and of the wide part were free from papillae and in JQ three transverse rows of papillae, one transverse mucosal fold were distributed around the choanal slit and caudally directed papillae were observed on their edges. By SEM in European magpie and Common raven spinous conical papillae lined up throughout the medial borders of the choanal cleft (Erdogan and Alan, 2012). Our findings in JQ similar to the statement of Erdoğan and Pérez (2015) in southern lapwing who stated that the transversal row

J. Vet. Anat. of conical papillae was observed between the rostral and caudal parts of the choanal cleft. The latter authors added that numerous, densely organized conical papillae also surrounded the entrance of the choanal cleft. In this respect, in chicken and goose, the rostral portion of the narrow part is devoid of papillae (Mohamed and Zayed, 2003). Functionally, the papillae organizing around choanal cleft obstruct escaping of foods into the cleft and the others facilitate the movement of nutrients into the esophagus (Nickle et al., 1977).

By scanning electron microscopy, in LD few small openings of palatine salivary glands were observed on each side of the narrow part of the choanal slit while in JQ numerous openings of the medial and lateral palatine salivary glands were scattered on both sides of the caudal narrow part of the choanal slit which increased caudally. In this connection, Madkour (2011) mentioned that several small openings of palatine salivary glands were recognized on each side of the caudal part of the median longitudinal ridge and on both sides of the choanal slit. However, Mohamed and Zayed (2003) mentioned in the chicken that, the rostral part of the palate has longitudinal arranged numerous openings (palatine) on both sides of the lateral palatine ridges. The latter authors 
conflict with our results in JQ that few palatine openings were observed on and lateral to the caudal part of the lateral palatine ridge.

The present investigation showed that the mucosa of the palate consisted of a highly cornified stratified squamous epithelium. The cornification increased in thickness laterally toward the beak edges as mentioned in duck (Madkour, 2011) and the mucosa of the palate of turkey becomes thickened and folded at the level of both medial and lateral palatine ridges (Sayed et al., 2016). The buccal cavity and esophagus are lined with cornified epithelium to resist the damage by hard food (Leake, 1975). However, in the chicken, the epithelium of the palate is not keratinized (King, 1975). In agreement with Soliman and Madkour (2017), they mentioned that oral mucosa of JQ was rich in Merkel corpuscles and Herbst corpuscles which were formed of fine collagen lamellae and some Herbst corpuscles had two axons. Corpuscles in the oropharynx are contributed in selection and transferring food (Crole and Soley, 2014). The palatine salivary glands on each side of choanal slit surrounded by smooth muscle fibers in the major salivary glands of adult poultry (Calhoun, 1954) are in agreement with our finding. The openings of each medial group of the palatine glands are

J. Vet. Anat. more numerous than those of the lateral group (King, 1975), was similar to that observed in the results of $J Q$ and contrary to the results of LD. Our works added that mucous glands are observed within the lamina propria of the median septum at the level of wide part of the choanal slit of JQ.

\section{References}

Abumandour, M. M. (2014): "Gross anatomical studies of the oropharyngeal cavity in Eurasian hobby (Falconinae: Falco Subbuteo, Linnaeus 1758)." Journal of Life Sciences Research 1(4): 80-92.

Adang, K. L., Ezealor A. U., Abdu, P. A. and Yoriyo, K. P. (2008). "Food habits of four sympatric columbids (Aves: Columbidae) in Zaria, Nigeria." Continental Journal of Biological Sciences 1: 1-9.

Ali, S. and Ripley, S. D. (1981). Han-dbook of the Birds of India and Pakistan. New Delhi: Oxford University Press.

Argyrous, G. (2005). Statistics for research; with a guide to SPSS. Sage, London.

Barilani, M., Derégnaucourt, S., Galle-go, S., Galli, L., Mucci, N., Piombo, R., Puigcerver, M., Rimondi, S., Rodrí-guez-Teijeiro, J. and Spanò, S. (2005). "Detecting Vol. 11, No. 2, (2018) 17 - 39 
hybridization in wild (Coturnix $c$. coturnix) and domesticated (Coturnix c. japonica) quail populations." Biolo-gical Conser-vation 126(4): 445-455.

Buchwalder, T. and Wechsler, B. (1997). "The effect of cover on the behaviour of Japanese quail (Coturnix japonica)." Applied Animal Behaviour Science 54(4): 335-343.

Calhoun, M. L. (1954). Microscopical anatomy of the digestive system of the chicken. Ames, lowa State College Press.

Crole, M. R. and Soley, J. T. (2011). "Distribution and structure of glandular tissue in the oropharynx and proximal esophagus of the emu (Dromaius novaehollandiae)." Acta Zoologica 92 (3): 206-215.

Crole, M. R. and Soley, J. T. (2014). "Comparative distribution and arrangement of Herbst corpuscles in the oropharynx of the ostrich (Struthio camelus) and emu (Dromaius novaehollandiae)." Anat Rec (Hoboken) 297(7): 1338-1348.

Erdogan, S. and Alan, A. (2012). "Gross anatomical and scanning electron microscopic studies of the oropharyngeal cavity in the European magpie (Pica pica) and the common raven (Corvus corax)."

J. Vet. Anat.
Microscopy research and technique 75(3): 379-387.

Erdoğan, S. and Pérez, W. (2015). "Anatomical and scanning electron microscopic characteristics of the oropharyngeal cavity (tongue, palate and laryngeal entrance) in the southern lapwing (Charadriidae: Vanellus chilensis, Molina 1782)." Acta Zoologica 96(2): 264-272.

Hassouna, E. (2002). "Morphyological studies on the phyarynged cavity of duck, Anas boushius domesticus." Assiut Veterinary Medical Journal (Egypt) 47(49): 2141.

Igwebuike, U. M. and Anagor, T. A. (2013). "The morphology of the oro-pharynx and tongue of the muscovy duck (Cairina moschata)." Veterinarski Arhiv 83(6): 685-693.

Jayachitra, S., Balasundaram, K., Iniyah, K., Sivagnanam, S. and Tamilselvan, S. (2015). "Morphology of oropharyngeal cavity in guinea fowl (Numida meleagris)." International Journal of Advanced Multidisciplinary Research IJAMR 2: 99-102.

King, A. S. (1975). Aves respiratory system. In: Getty, R. (ed.). Sisson and Grossman's the anatomy of the domestic animals. W. B. Saunders Co., Philadelphia and London. 
King, A. S. and McLelland, J. (1984). Birds, their structure and function, Bailliere Tindall, $1 \mathrm{St}$. Annes Road.

Leake, L. D. (1975). Comparative histology. Academic press, London, New York., Asubsidiary of Haracourt Brace Fovanovich.

Madkour, F. A. M. (2011). Some de-velopmental studies on the roof of the oropharynx of the duck. . South Valley University, Egypt. M.V.Sc. .

Mohamed, S. and Zayed, A. (2003). "Gross anatomical and scanning electron microscopical studies on palate of some birds." Assiut Veterinary Medecine Journal 49: 1-17.

Moussa, E. A. and Hassan, S. A. (2013). "Comparative gross and surface morphology of the oropharynx of the hooded crow (Corvus cornix) and the cattle egret (BubuIcus ibis)." Journal of Veterinary Anatomy 6(1): 1-15.

Nickle, R., Schummer, A. and SEIFERLE, E. (1977). "Anatomy of the domestic bird." Translated by SILLER, WG and PA WIGHT. Verlag, Paul Parey, Berlin: 72-75.

Pappas, J. (2013). "'"Coturnix japonica". Animal Diversity Web. ."
Puigcerver, M., Vinyoles, D. and Rodríguez-Teijeiro, J. D. (2007). "Does restocking with Japanese quail or hybrids affect native populations of common quail Coturnix coturnix?" Biological Conservation 136(4): 628-635.

Rodrigues, M. N., Tivane, C. N., Carvalho, R. C., Oliveira, G. B., Silva, R. S., Ambrosio, C. E., Oliveira, M. F. and Miglino, M. A. (2012). "Gross morphology of rhea oropharyngeal cavity." Pesquisa Veterinária Brasileira 32: 53-59.

Rossi, J. R., Baraldi-Artoni, S. M., Oliveira, D., Cruz, C. d., Franzo, V. S. and Sagula, A. (2005). "Morphology of beak and tongue of partrigde Rhynchotus rufescens." Ciência Rural 35(5): 1098-1102.

Satheesan, S. M., Prakash, R. and Datye, H. (1990). "Biometrics and food of some doves of the genus Strepto-pelia." Journal of the Bombay Natural History Society 87 : 452453.

Sayed, R. K., Abdalla, K. E., Ahmed, A. K. and Saleh, A. M. (2016A). "Palate of Turkey (Meleagris gallopavo): Gross Anatomical, Light and Scanning Electron Microscopical Study." Journal of Advanced Veterinary Research 6(3): 81-88. 
Sayed, R. K., Saleh, A. M., Ahmed, A. K. and Abdalla, K. E. (2016). "Gross Anatomical, Light and Scanning Electron Microscopic Studies on the Pharyngeal Roof of Turkey (Meleagris gallopavo): Comparative Study." Journal of Advanced Veterinary Research 6(4): 112-117.

Soliman, S. A. and Madkour, F. A. (2017). "A comparative analysis of the organization of the sensory units in the beak of duck and quail." Histol Cytol Embryol 1(4): 1-16.

Squier, C. A. and Kremer, M. J. (2001). "Biology of oral mucosa and esophagus." J. Nalt. Cancer Inst Monoger 29: 7-15.

Sundaram, V., Nissar, S., Arthanari, K., Basha, S. and Ramesh, G. (2015). "Functional morphology of the epidermal structures of the feeding apparatus of Guinea fowl (Numidia melea-gris)." International Journal of Advanced Research 3 ( 10): 1601 - 1608.

Ticehurst, C. B. (1923). "The Birds of Sind. (Part V.)." Ibis 65(3): 438473.

Tivane, C., Rodrigues, M. N., Soley, J. T. and Groenwald, H. B. (2011). "Gross anatomical features of the oropharyngeal cavity of the ostrich (Struthio camelus)." Pesquisa Veterinária Brasileira 31(6): 543-550.
Ziswiler, V. (1965). "Zur Kenntnis des Samenöffnens und der Struktur des hörnernen Gaumens bei körnerfressenden Oscines." Journal of Ornithology 106(1): 1-48.

\section{Corresponding author:}

Fatma A. Madkour

Telefax: +20965211223

E-mail: madkour.fatma@yahoo.com madkour.f@vet.svu.edu.eg Orchid Number: 0000000356419260 

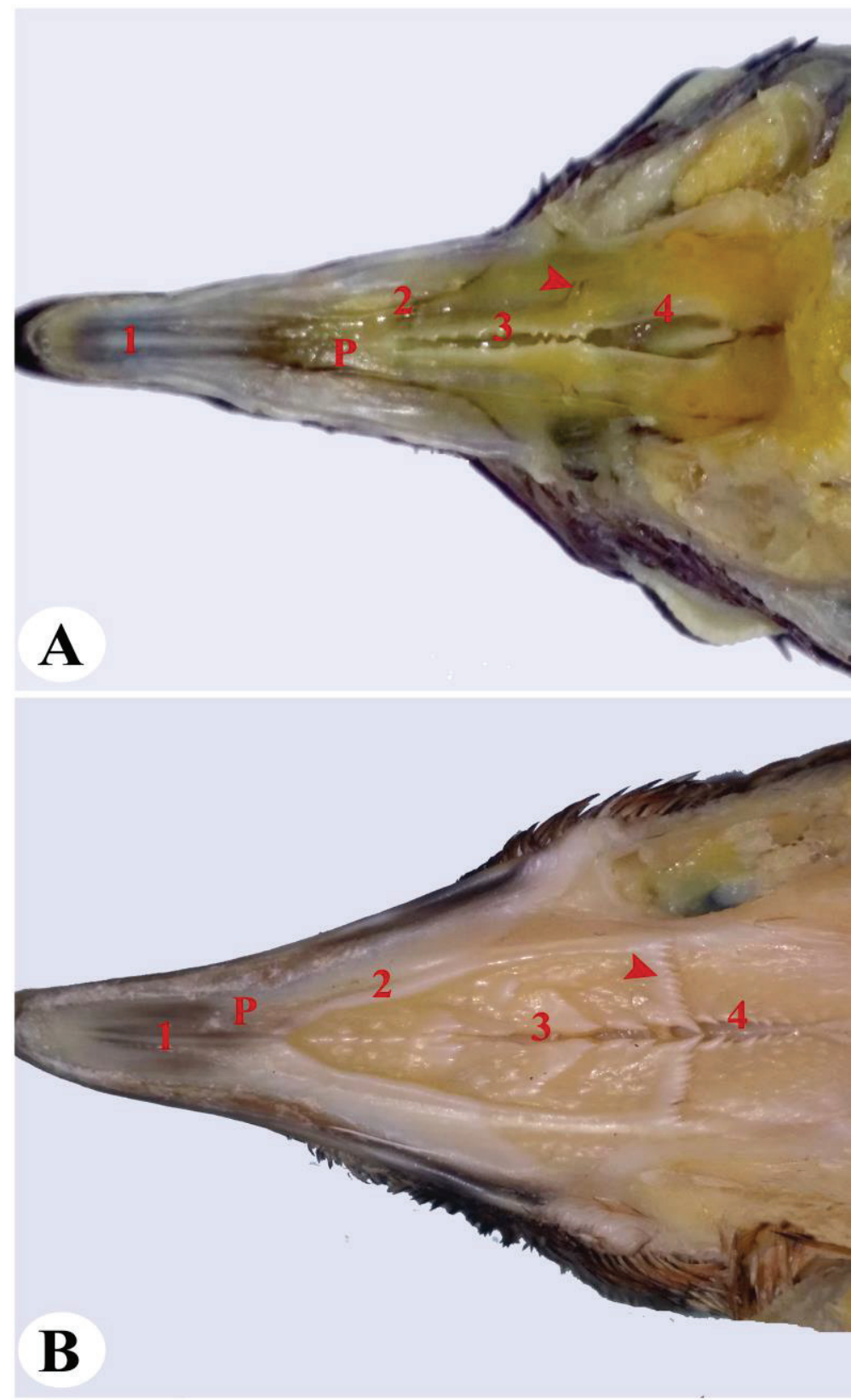

Fig (1): Photograph of the roof of the oropharynx of laughing dove (A) and of Japanese quail $(B)$. Note, palate $(P)$, 1-median palatine ridge, 2-lateral palatine ridge, 3- narrow part of choanal slit, 4-wide part of choanal slit, the line of demarcation between 2 parts marked by caudally directed papillae (arrow head). 


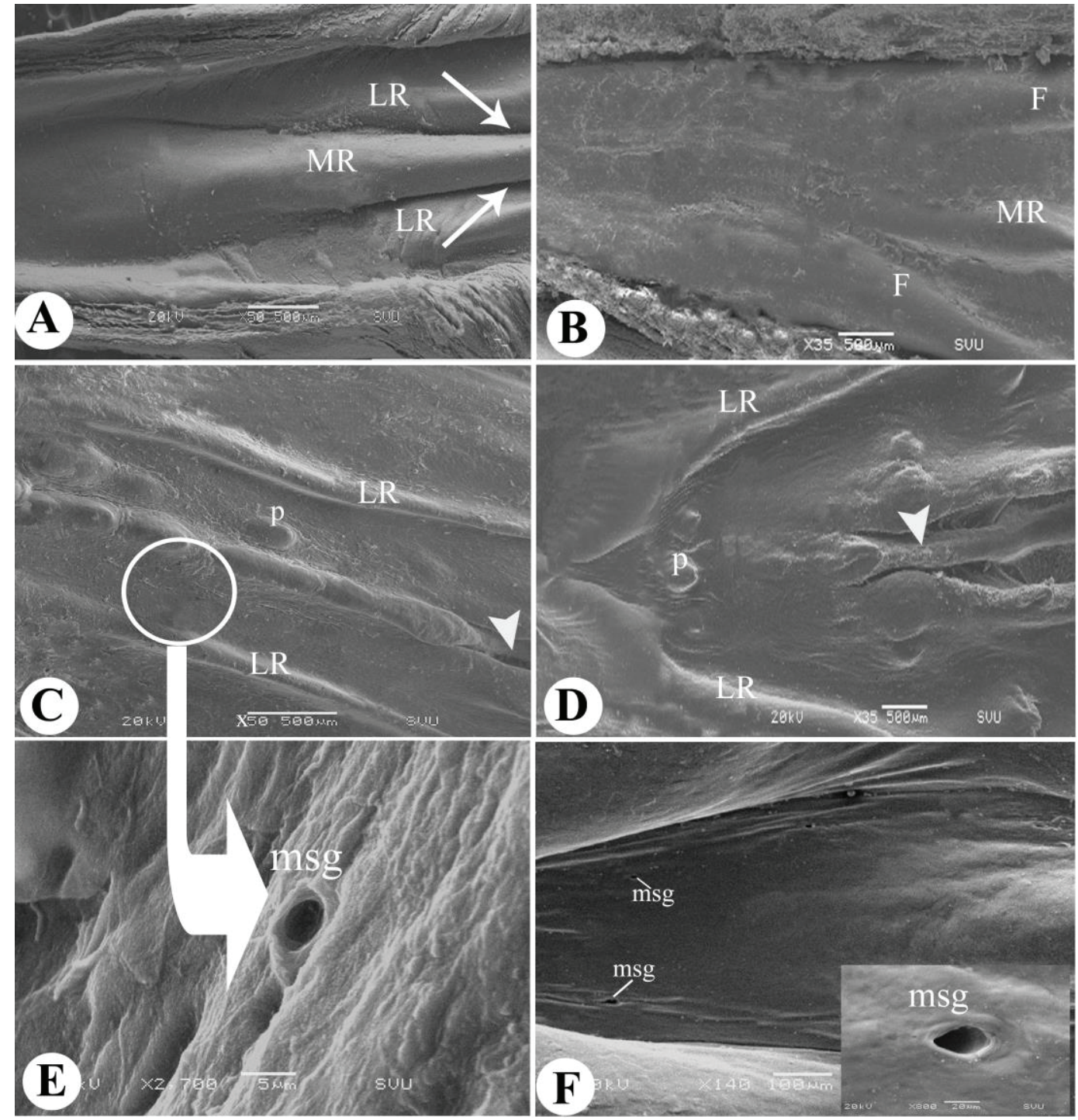

Fig (2): Scanning electron micrograph of the palate of laughing dove (A, C \& E) and of Japanese quail (B, D\&F). Note, median ridge (MR), lateral ridges (LR), shallow grooves (arrows), mucosal fold $(F)$, median septum (arrow head), papillae $(P)$ and openings of maxillary salivary glands (msg). 


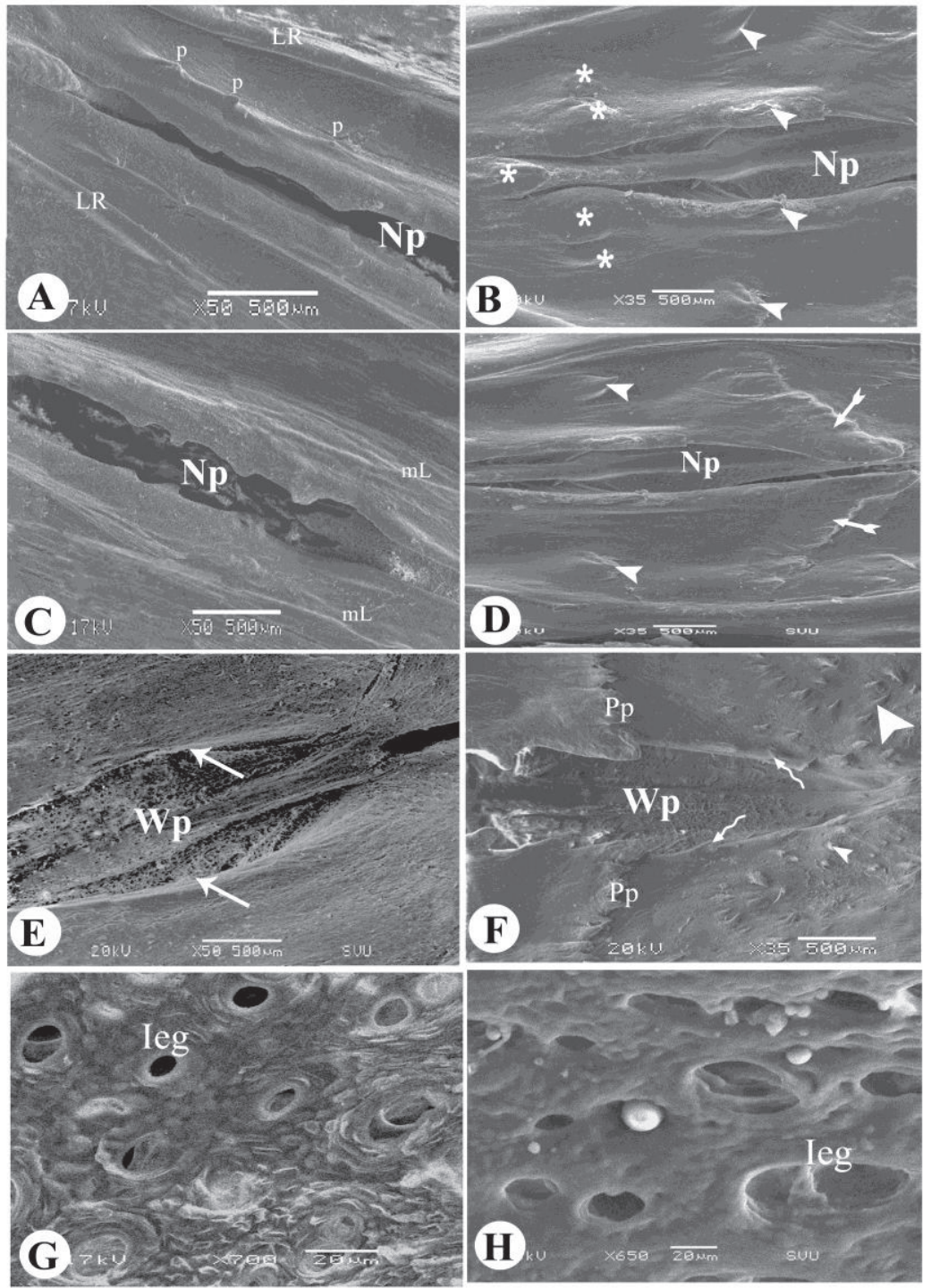

Fig (3): Scanning electron micrograph of the choanal slit of laughing dove (A, C, E\&G) and of Japanese quail (B, D, F\& H). Note, narrow (Np) and wide (Wp) parts of choanal slit, lateral ridges (LR), 3caudally directed papillae $(P)$ laterally to the edges of the narrow parts, mucosal elevation $(\mathrm{mL})$ on each side of the caudal half of the narrow part, first row (stars), second row (arrow heads) of papillae, wing- shaped mucosal fold (barbed arrows), $V$-shaped papillae (Pp) at junction between the narrow and wide parts of the choanal slit, smooth edges of the wide part of the slit (arrows), papillae on the edges of the wide part (twisted arrows), conical shaped papillae (large arrow head), nodular shaped papillae (small arrow head), and openings of the intraepithelial glands (leg). 


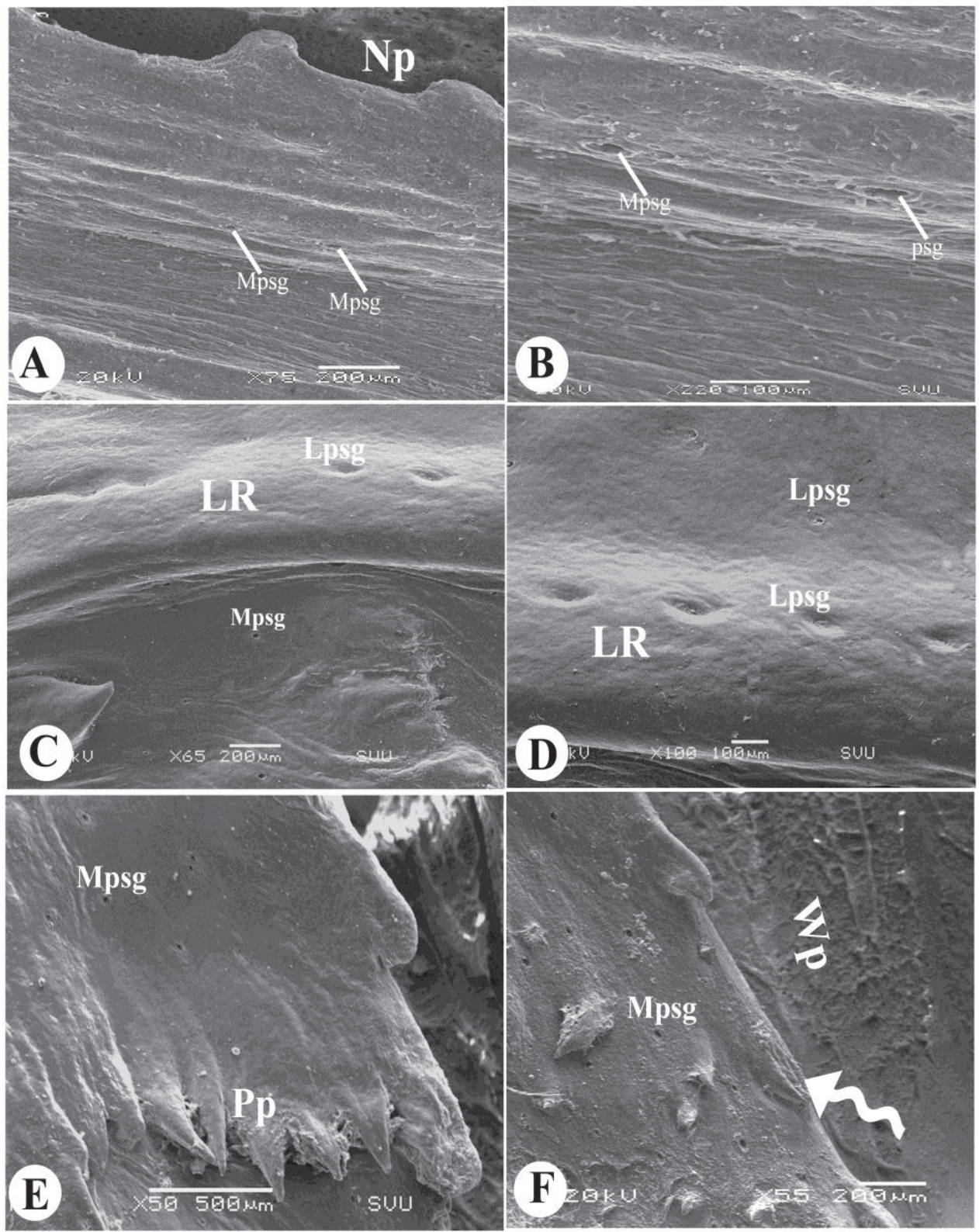

Fig (4): Scanning electron micrograph of the palate around the choanal slit of laughing dove $(A, B)$ and of Japanese quail $(C-F)$. Note, openings of the medial palatine salivary glands (Mpsg) on each side of the narrow (Np) and wide (Wp) parts of the choanal slit, lateral palatine salivary glands (Lpsg) on and lateral to the lateral ridges (LR), V-shaped papillae (Pp) at junction between the narrow and wide parts of the slit, papillae (twisted arrow) on the edges of the wide part. 


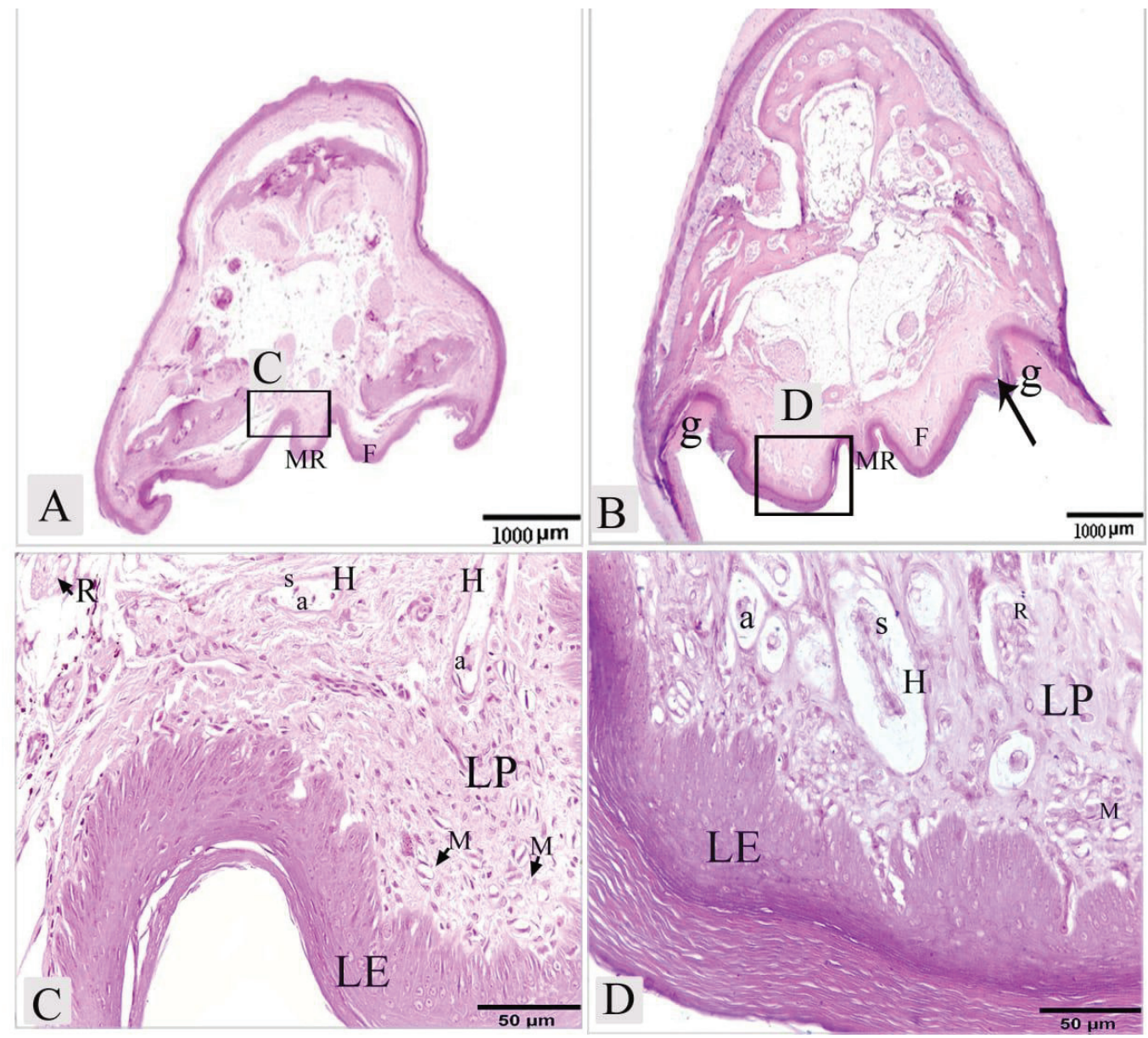

Fig (5): Photomicrograph of a cross-section of the rostral part of the palate of laughing dove $(A \& C)$, of Japanese Quail (B\&D) stained with H\&E. A\&B: Note, a median palatine ridge (MR) with 2 folds on each sides $(F)$, another 2folds (arrow) separated laterally from the beak edges by grooves (g). B\&D: Note, cornified lamina epithelialis (LE), lamina propria (LP) contained Herbst corpuscles (H) consisted of axon (a), sensory cells (S), Ruffini (R) and Merkel (M) corpuscles. 

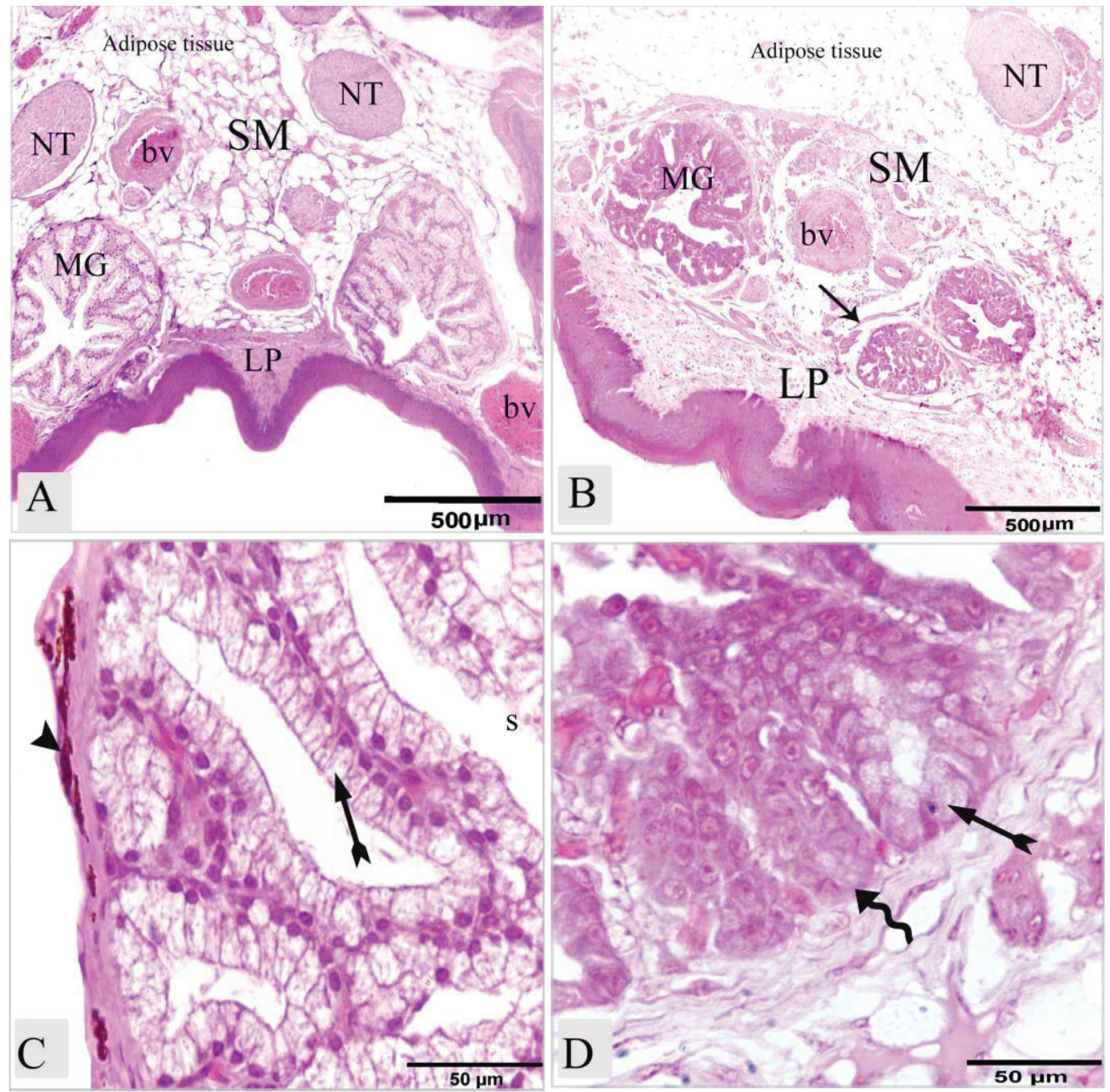

Fig (6): Photomicrograph of cross-section of rostral part of the palate at level of the maxillary glands of laughing dove (A\&C), of Japanese Quail (B\&D) stained with H\&E. Note, lamina propria (LP), submucosa (SM) consisted of nerve trunk (NT), blood vessels (bv), maxillary glands (MG) lining by mucous secretory cells (barbed arrow), serous secretory cells (twisted arrow) surrounding by thin layer of smooth muscles fibers (arrow), melanocytes (arrowhead) and abundant adipose tissue (adipose tissue) caudal to glands. 


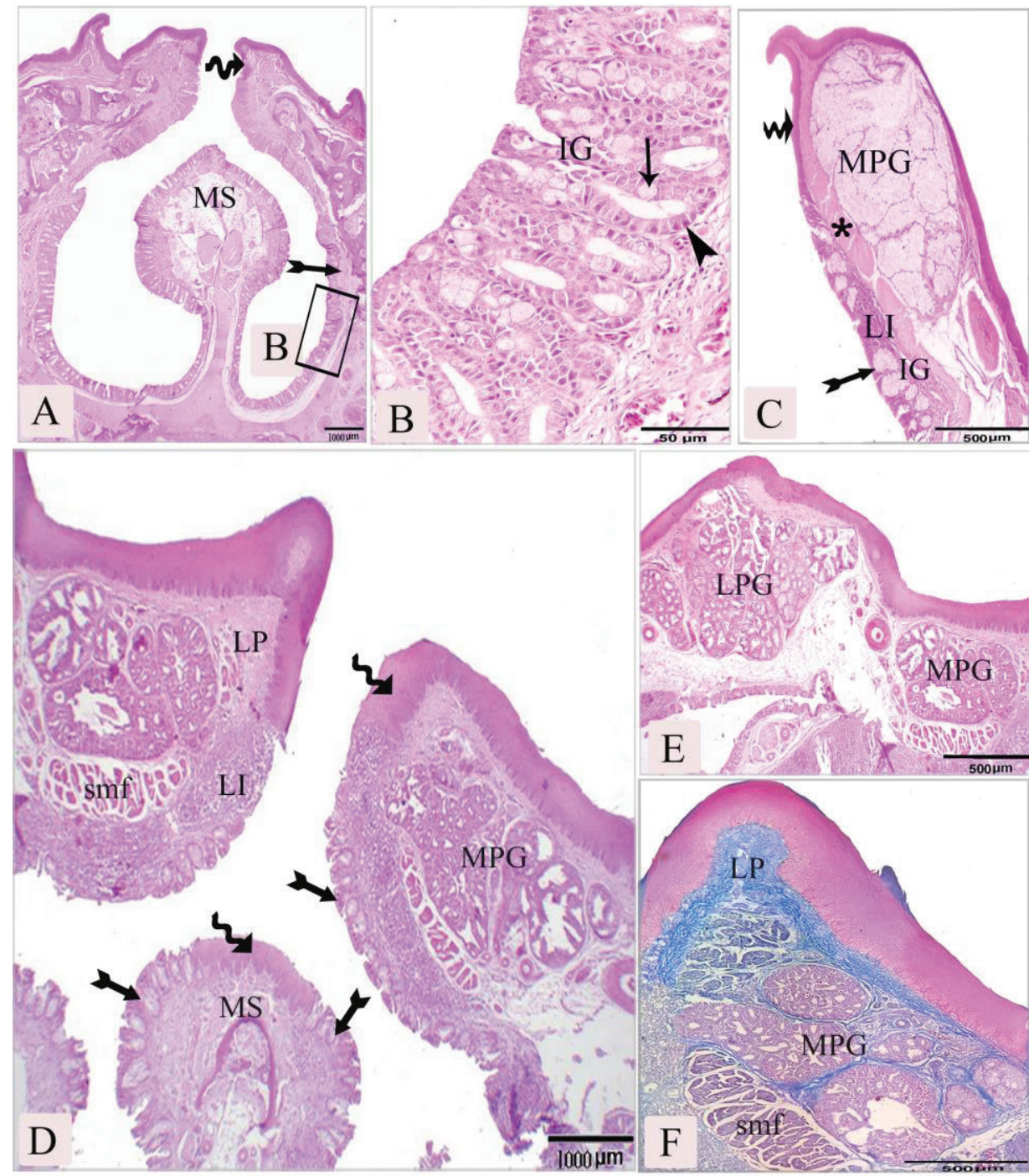

Fig (7): Photomicrograph of the narrow part of the choanal slit of laughing dove (A-C), of Japanese quail (D-F) stained with H\&E, (F) stained with Masson trichrome. Note, transforming of cornified stratified squamous epithelium (twisted arrow) into pseudostratified ciliated columnar epithelium (barbed arrow), respiratory epithelium interrupted by intraepithelial glands(IG) which were seromucoid type; mucous secretory cells (arrow) and serous secretory cells (arrow head), lamina propria (LP) with excessive lymphatic infiltration (LI) around intraepithelial glands(IG), medial (MPG) and lateral (LPG) palatine glands surrounded by smooth muscle fibers (smf) and thick collagen fibers (star) and median septum (MS) lined by respiratory epithelium (barbed arrow) and stratified squamous epithelium (twisted arrow) at the center. 


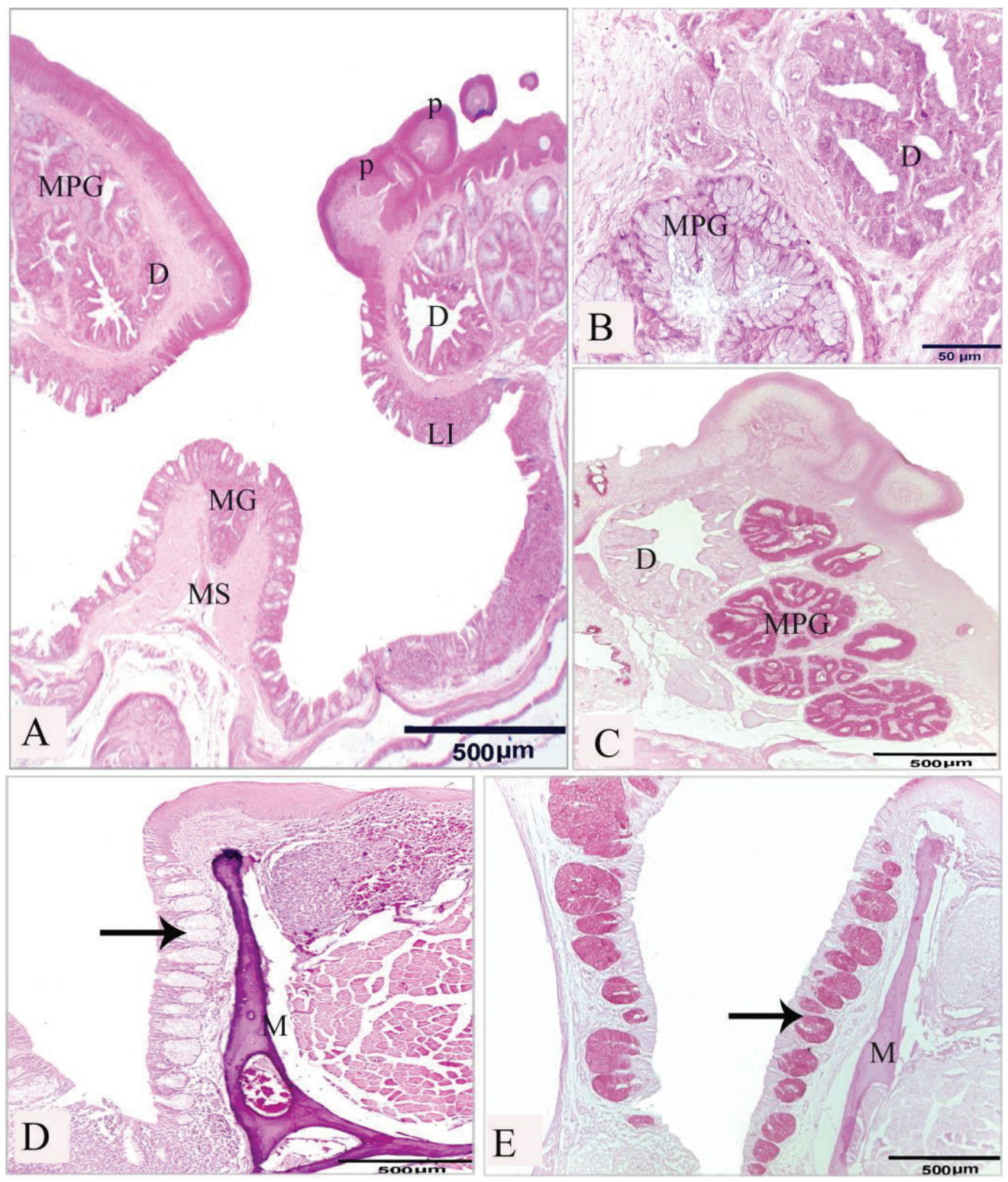

Fig (8): Photomicrograph of the wide part of the choanal slit of Japanese quail (A-C), of laughing dove (D\&E). A, B, D stained with $\mathrm{H} \& \mathrm{E},(\mathrm{C}, \mathrm{E})$ stained with PAS. Note, variable sizes of papillae $(P)$ consisted of core of connective tissue covered with highly keratinized stratified squamous epithelium, compound tubuloalveolar medial palatine glands (MPG) showed positive PAS reactions, their ducts (D) lining by cuboidal cells showed negative PAS reactions, mucous glands (MG) were observed within lamina propria of the median septum (MS), the mucous intraepithelial glands showed positive PAS reaction (arrow), plate of the maxillary bone $(\mathrm{M})$ at submucosa. 


\section{Animal species in this Issue}

\section{Common quail, (Coturnix coturnix)}

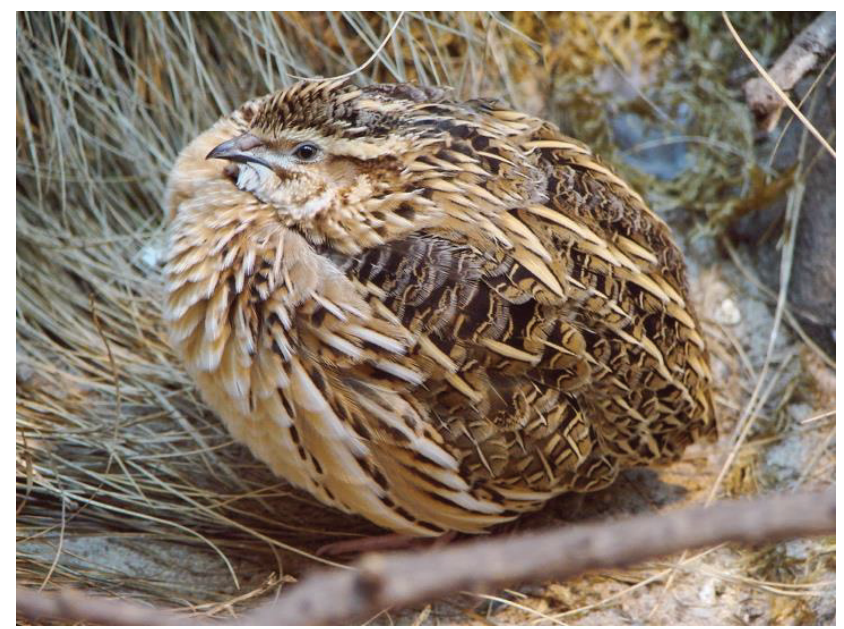

Kingdom: Animalia \& Phylum: Chordata \& Class: Aves \& Subclass: Neomithes \& Infraclass: Neognathae \& Superorder: Neoaves \& Order: Pelecaniformes \& Family: Ardeidae \& Genus: Ardea \& Species: A. cinerea

It is a small, round bird, essentially streaked brown with a white eyestripe, and, in the male, a white chin. As befits its migratory nature, it has long wings, unlike the typically short-winged gamebirds. It measures roughly $18.0-21.9 \mathrm{~cm}$ and weighs $91-131 \mathrm{~g}$.

This is a terrestrial species, feeding on seeds and insects on the ground. It is notoriously difficult to see, keeping hidden in crops, and reluctant to fly, preferring to creep away instead. Even when flushed, it keeps low and soon drops back into cover. Often the only indication of its presence is the distinctive "wetmy-lips" repetitive song of the male. The call is uttered mostly in the mornings, evenings and sometimes at night. It is a strongly migratory bird, unlike most game birds.

Upon attaining an age of 6-8 weeks, this quail breeds on open arable far land and grassland across most of Europe and Asia, laying 6-12 eggs in a ground nest. The eggs take from 16-18 days to hatch.

Source: Wikipedia, the free encyclopaedia 